\title{
Optimization of white matter tractography for pre-surgical planning and image-guided surgery
}

\author{
KONSTANTINOS ARFANAKIS ${ }^{1,2}$, MINZHI GUI ${ }^{1}$ and MARIANA LAZAR ${ }^{3}$ \\ ${ }^{1}$ Department of Biomedical Engineering, Illinois Institute of Technology; ${ }^{2}$ Brain Research Imaging Center, \\ The University of Chicago, Chicago, IL; ${ }^{3}$ Keck Laboratory for Functional Brain Imaging and Behavior, \\ University of Wisconsin-Madison, Madison, WI, USA
}

Received July 7, 2005; Accepted September 27, 2005

\begin{abstract}
Accurate localization of white matter fiber tracts in relation to brain tumors is a goal of critical importance to the neurosurgical community. White matter fiber tractography by means of diffusion tensor magnetic resonance imaging (DTI) is the only non-invasive method that can provide estimates of brain connectivity. However, conventional tractography methods are based on data acquisition techniques that suffer from image distortions and artifacts. Thus, a large percentage of white matter fiber bundles are distorted, and/or terminated early, while others are completely undetected. This severely limits the potential of fiber tractography in pre-surgical planning and image-guided surgery. In contrast, TurbopropDTI is a technique that provides images with significantly fewer distortions and artifacts than conventional DTI data acquisition methods. The purpose of this study was to evaluate fiber tracking results obtained from Turboprop-DTI data. It was demonstrated that Turboprop may be a more appropriate DTI data acquisition technique for tracing white matter fibers than conventional DTI methods, especially in applications such as pre-surgical planning and image-guided surgery.
\end{abstract}

\section{Introduction}

Fiber tractography (1) is a non-invasive method that uses white matter fiber orientation information obtained with diffusion tensor imaging (DTI) (2) in order to produce estimates of fiber pathways that connect different brain regions. Tractography is gradually becoming an important tool for clinical applications, such as pre-surgical planning, where knowledge of the exact location of the lesion with respect to eloquent white matter pathways is of great

Correspondence to: Dr Konstantinos Arfanakis, Department of Biomedical Engineering, Illinois Institute of Technology, 10 West 32nd Street, E1-116, Chicago, IL 60616, USA

E-mail: arfanakis@iit.edu

Key words: diffusion tensor imaging, tractography, tumor, pre-surgical planning, image-guided surgery importance. Fiber tracking has been used to map the corticospinal tract, subcortical pathways serving cortical language sites, and the superior longitudinal fasciculus in patients with brain tumors or other space occupying lesions located in the vicinity of these fiber tracts (3-5). In all cases, tractography provided helpful information when planning the appropriate surgical strategy.

The accuracy and reproducibility of fiber tracking results depend heavily on the noise level and amount of artifacts of the DTI data used. However, conventional tractography is performed based on DTI data acquired using diffusion sequences with an echo-planar (EPI) signal readout (6). These acquisitions suffer from image distortions, signal loss and pile-up near regions with magnetic field inhomogeneities, such as those at tissue-air and tissue-bone interfaces (7). Furthermore, EPI-based DTI is sensitive to distortions and artifacts due to eddy currents (8). In contrast, Turboprop imaging is an MRI data acquisition and reconstruction technique with greatly reduced sensitivity to various sources of image artifacts (9-11). Turboprop images contain significantly fewer field-inhomogeneity-related artifacts than those in EPI, and are immune to warping due to eddy currents (10). Also, multiple measurements of the low spatial frequency information performed for each Turboprop image allow correction of the rotation and translation of the subject, and reduction of motion artifacts.

Because of the reduced sensitivity of Turboprop-DTI to various sources of image artifacts, the purpose of this work was to evaluate Turboprop as a DTI data acquisition technique for use in tractography. Our hypothesis was that fiber bundles mapped from Turboprop-DTI data would be undistorted and in agreement with the anatomy, even in regions near significant magnetic field inhomogeneities. If our hypothesis is valid, then Turboprop-DTI may significantly enhance the role of tractography in pre-surgical planning.

\section{Materials and methods}

Scans were performed on patients with brain tumors and arteriovenous malformations (AVMs), as well as on healthy controls, on a $1.5 \mathrm{~T}$ and a 3T GE Signa MRI scanner (Waukesha, WI) equipped with high-speed gradients (40 $\mathrm{mT} / \mathrm{m}$ maximum amplitude and $150 \mathrm{mT} / \mathrm{m} / \mathrm{msec}$ slew rate). All subjects gave their signed consent in accordance 


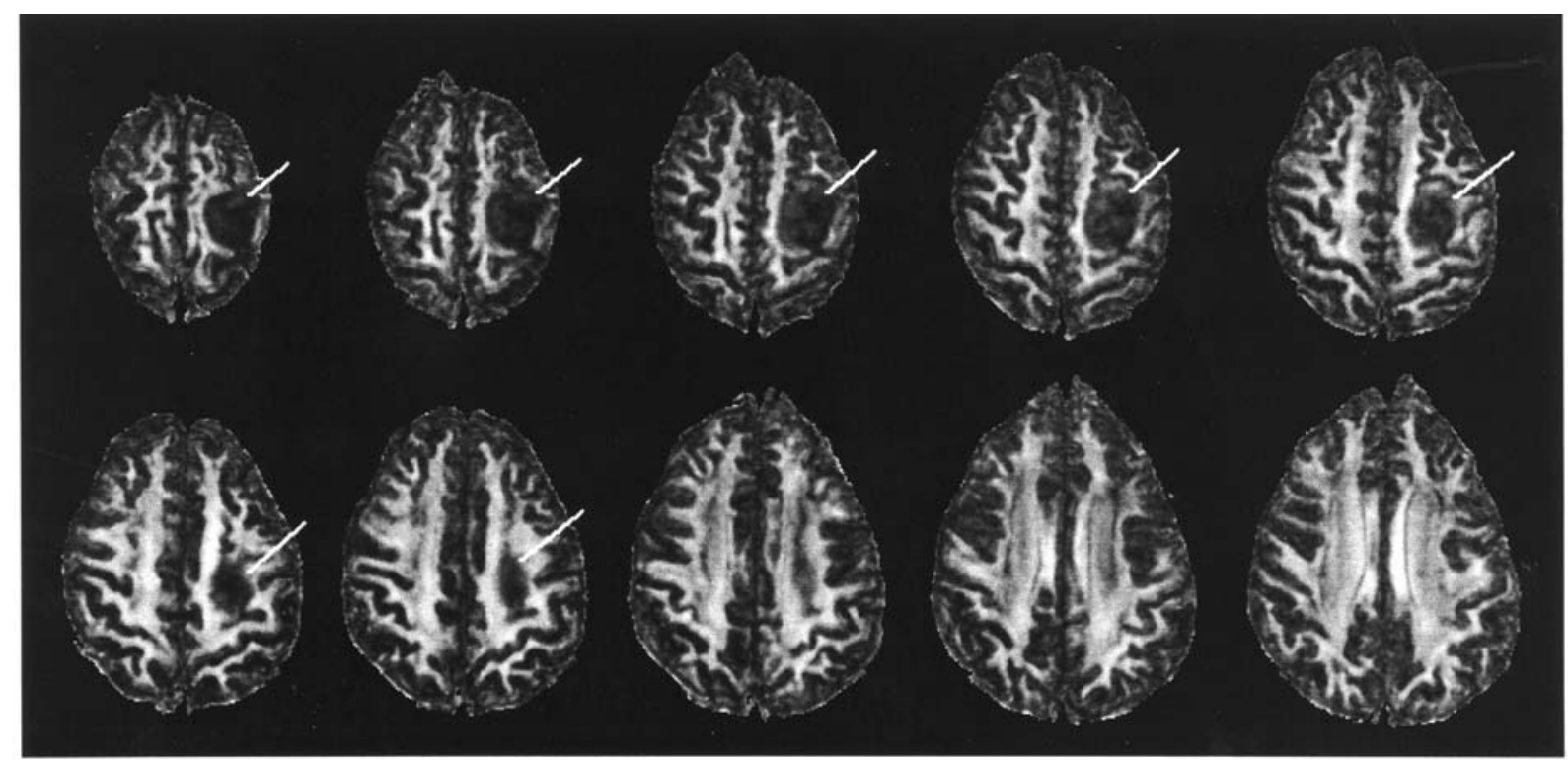

Figure 1. Diffusion anisotropy maps from 10 consecutive axial slices of a patient with a tumor in the left parietal lobe (left is on the right side of the image). The arrows show the location of the tumor. The lesion appears to have displaced normal fiber tracts and shifted the midline.

with institutional policies. Patients were scanned with conventional spin-echo EPI-DTI (SE-EPI-DTI), and healthy controls with Turboprop-DTI. The imaging parameters for SE-EPI-DTI were: $\mathrm{TR}=5400 \mathrm{~ms}, \mathrm{TE}=71.8 \mathrm{~ms}$, field of view $=24 \times 24 \mathrm{~cm}, 36$ contiguous axial slices, $3 \mathrm{~mm}$ slice thickness, and the phase encoding direction was anterior-posterior. The amplitude of the total diffusion gradient was $40 \mathrm{mT} / \mathrm{m}$ and applied in 12 non-collinear directions uniformly distributed in a three-dimensional space. The effective diffusion weighting was $b=900 \mathrm{sec} / \mathrm{mm}^{2}$. T2 images with no diffusion weighting $\left(b=0 \mathrm{sec} / \mathrm{mm}^{2}\right.$ ) were also acquired at the beginning of each DTI scan. Acquisition of all $b=900 \mathrm{sec} / \mathrm{mm}^{2}$ and $b=0 \mathrm{sec} / \mathrm{mm}^{2}$ images was repeated 5 and 10 times, respectively. The duration of the SE-EPI-DTI scan was 6 min and $18 \mathrm{sec}$. Eddy-currentinduced distortions were corrected in the images acquired with SE-EPI-DTI by registering all diffusion weighted (DW) images to the mean DW image for each slice, using a twodimensional, perspective, 8-parameter registration algorithm (12).

The imaging parameters for Turboprop-DTI were: TR = $5000 \mathrm{~ms}, 8$ spin-echoes per TR $(\mathrm{ETL}=8), 5 \mathrm{k}$-space lines per spin-echo (turbo factor $=5$ ), thus each $\mathrm{k}$-space blade contained $8 \times 5=40$ lines, $16 \mathrm{k}$-space blades per image, field of view $=24 \times 24 \mathrm{~cm}, 36$ contiguous axial slices, and a $3-\mathrm{mm}$ slice thickness. DW images with $b=900 \mathrm{sec} / \mathrm{mm}^{2}$ were acquired for the same 12 diffusion directions as in SE-EPIDTI. Two $b=0 \mathrm{sec} / \mathrm{mm}^{2}$ images were also acquired. The duration of the Turboprop-DTI scan was $18 \mathrm{~min}$ and $55 \mathrm{sec}$. The 36 axial slices used in both DTI sequences covered most of the brain. The diffusion tensors were estimated from the DTI data in each voxel. Maps of fractional anisotropy (FA), which characterizes diffusion anisotropy and the anisotropy of the fiber structure, were produced for all slices. In these maps, healthy white matter tissue is characterized by high signal intensities due to highly anisotropic diffusion. Grey matter, cerebrospinal fluid (CSF) and structurally damaged white matter are characterized by low intensities, due to the more isotropic nature of diffusion in these tissues. High resolution anatomical data, with minimum distortions, were acquired in each subject and compared to the results from SE-EPI-DTI and Turboprop-DTI in order to assess the amount of distortions in the two DTI sequences.

Regions of interest (ROI) were selected in all subjects, which were used as seeds for fiber tracking. These ROIs enclosed fibers from the following fiber bundles: cingulum, fornix, corpus-callosum (CC), forceps minor, corticospinal tract (CST) and corona radiata, inferior and superior longitudinal fasciculi (ILF, SLF), anterior commisure (AC), and inferior occipito-frontal fasciculi (IOF). Tractography results were compared between different acquisition techniques in terms of distortions and similarities to known fiber tract anatomy.

\section{Results}

Fig. 1 shows diffusion anisotropy maps from a patient with a brain tumor in the left parietal lobe. The tumor shown in Fig. 1 displaced normal fiber tracts and was located next to several major fiber pathways, such as the CST. Fig. 2 shows the tumor in red, and neighboring fibers (primarily fibers that connect the two hemispheres) wrapping around the lesion, demonstrating the significance of accurate fiber tracking. SE-EPI-DTI was used for data acquisition.

Comparison of the high-resolution anatomical data with the $b=0 \mathrm{sec} / \mathrm{mm}^{2}$ images obtained with SE-EPI-DTI revealed significant distortions, signal loss and pile-up, in the frontal and temporal lobes and the brainstem in SE-EPI-DTI. Fig. 3 shows high resolution anatomical (Fig. 3A) and $b=0 \mathrm{sec} / \mathrm{mm}^{2}$ DTI images (Fig. 3B) from a patient with an AVM in the left temporal lobe. Significant distortions can be seen in the temporal lobes and brainstem. Fibers of the left ILF, which is in the vicinity of the AVM, appeared to be interrupted, and most of them terminated in the lateral wall of the temporal lobe (Fig. 3C). The fibers did not reach the temporal pole as expected from anatomical studies and our previous fiber tracking experience. A coronal FA map (Fig. 3D) shows 


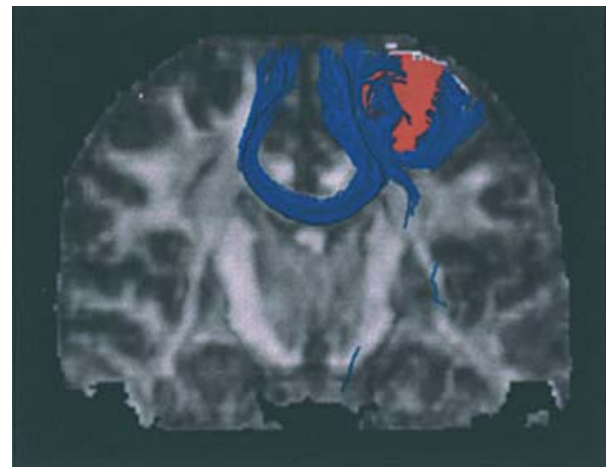

Figure 2. This image shows a coronal view of normal white matter fibers (blue lines) located in the vicinity of a tumor (red). The fibers appear to wrap around the tumor, which demonstrates the need for accurate tracking methods for use in pre-surgical planning. The greyscale image in the background is an FA map of a coronal slice from the patient. that the ILF fibers (blue) were near a region with signal pileup (arrow) caused by susceptibility artifacts. In an attempt to clarify if the fibers did not reach the temporal pole because of the AVM or due to other causes, fibers of the ILF in the right hemisphere were also traced. Although the right temporal lobe appeared healthy, the ILF fibers did not reach the temporal pole due to the severe distortions and artifacts (Fig. 3E). Therefore, comparison of the left and right ILF fibers was not conclusive as to why the left hemisphere ILF fibers did not reach the temporal pole, due to the distortions that were present in both temporal lobes.

Overlaying edge-maps produced from the anatomical images, onto FA maps from Turboprop-DTI demonstrated that the Turboprop-DTI data were in excellent agreement with the anatomy, even in regions near significant magnetic field inhomogeneities. Fibers of the cingulum, fornix, CC,

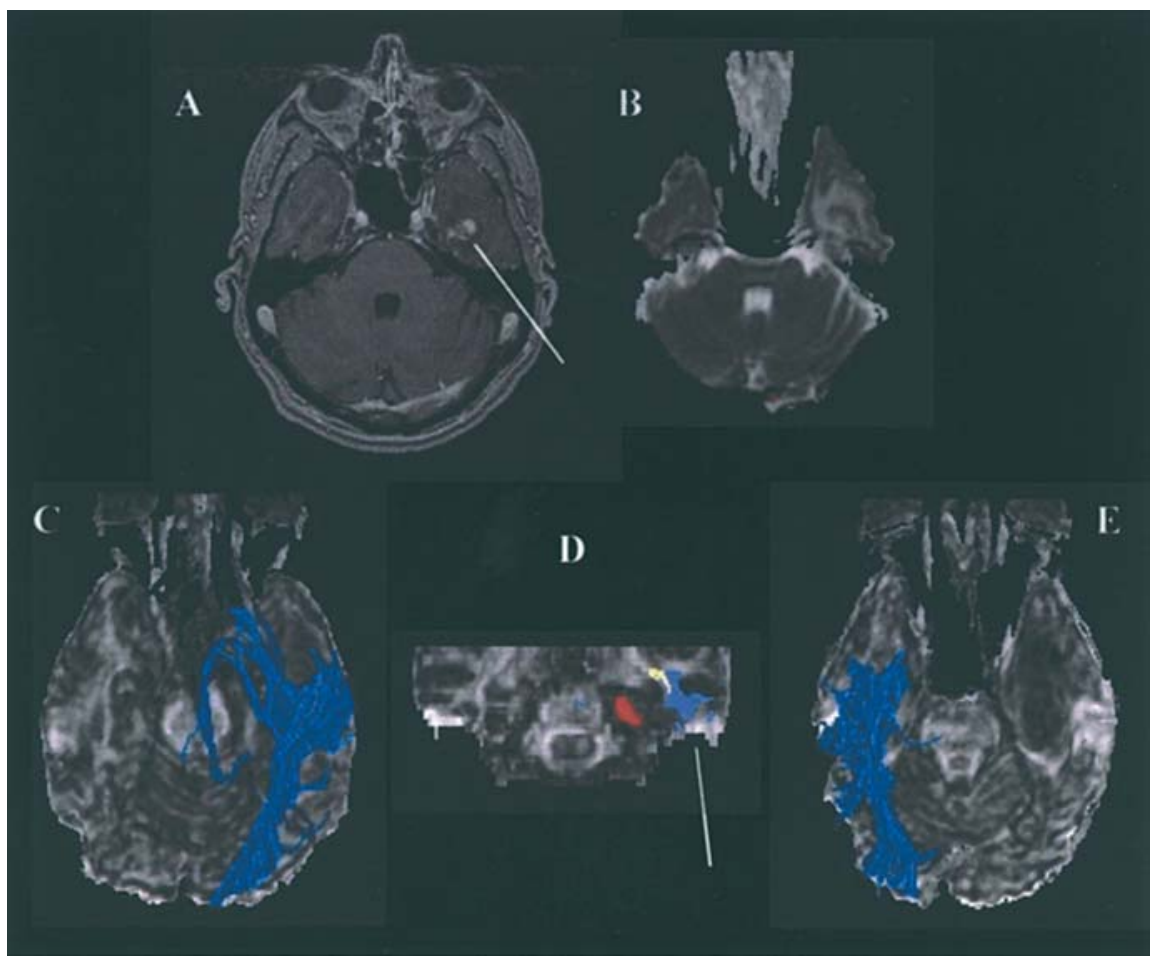

Figure 3. (A) High-resolution anatomical image from a patient with an AVM in the left temporal lobe shown with an arrow. (B) T2 image $\left(\mathrm{b}\right.$-value $\left.=0 \mathrm{sec} / \mathrm{mm}^{2}\right)$ of the same slice acquired with SE-EPI-DTI. There are visible distortions in the temporal lobes and the brainstem. (C) Axial projection of the left ILF fibers, which are in the vicinity of the AVM. Some of the fibers appear to be interrupted, and most of the remaining fibers end in the lateral wall of the temporal lobe. The fibers do not reach the temporal pole probably due to the AVM, image distortions, and noise. (D) A coronal FA map shows that the ILF fibers (blue) are near a region with signal pile-up (arrow) caused by susceptibility artifacts. (E) Axial projection of the right ILF fibers. Although the right temporal lobe appears healthy, the fibers do not reach the temporal pole due to the severe distortions and artifacts. Therefore, comparison of the left and right ILF fibers is not conclusive as to why the left ILF fibers did not reach the temporal pole.
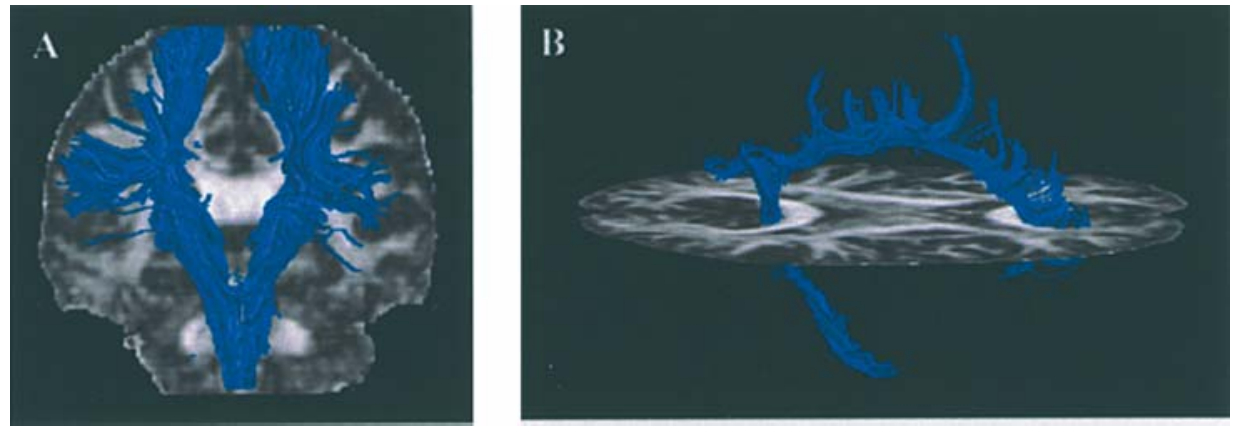

Figure 4. Fibers of the CST and corona radiata (A) and the cingulum (B), reconstructed from Turboprop-DTI data. 
U-fibers of the frontal lobe, CST and corona radiata, ILF, SLF, AC and IOF were successfully mapped (Fig. 4). All fibers traced from Turboprop-DTI data were determined to be undistorted and anatomically accurate representations of the corresponding fiber pathways.

\section{Discussion}

Conventional DTI data acquisition methods suffer severe image distortions and artifacts that may significantly reduce the accuracy of tractography results. Since precise mapping of white matter fiber tracts in relation to brain lesions is a goal of critical importance for pre-surgical planning, an alternative DTI data acquisition method is required. TurbopropDTI is a data acquisition and reconstruction technique with reduced sensitivity to a number of sources of image artifacts. In this study, it was shown that Turboprop-DTI data can be used to obtain undistorted, anatomically accurate, estimates of white matter fiber connections for use in pre-surgical planning and image-guided surgery.

Several variations of the SE-EPI-DTI pulse sequence, as well as post-processing algorithms, have been developed in order to reduce the effects of magnetic field inhomogeneities (7,13-15) and eddy currents $(8,16,17)$. However, all of these techniques increase the total scan time or are computationally intensive, or both, and none of them completely corrects susceptibility and eddy-current-related artifacts. Residual distortions and artifacts in DTI data obtained with SE-EPIDTI acquisitions may introduce errors in the tractography results.

Turboprop-DTI data acquisitions are generally slower than conventional SE-EPI-DTI acquisitions. In this study, Turboprop-DTI data were obtained 3 times slower than the SE-EPI-DTI data, and the signal-to-noise ratio (SNR) in the Turboprop-DTI data was lower than that in SE-EPI-DTI. To make Turboprop-DTI even more appropriate for clinical use, the acquisition time must be reduced to approximately 10-15 min, which may be possible by using k-space undersampling (18) or parallel imaging (19), but the reduction of acquisition time will lead to a decrease in SNR. Further studies are necessary to optimize the Turboprop-DTI acquisition and reconstruction, in order to compensate for the loss of SNR when accelerating data acquisition.

\section{Acknowledgements}

This study was supported in part by a grant from the Education and Research Initiative Fund (ERIF). The authors would like to thank Dr Aaron Field for providing the AVM patient data.

\section{References}

1. Basser PJ, Pajevic S, Pierpaoli C, Duda J and Aldroubi A: In vivo tractography using DT-MRI data. Magn Reson Med 44: 625-632, 2000.

2. Basser PJ and Pierpaoli C: Microstructural and physiological features of tissues elucidated by quantitative-diffusion-tensor MRI. J Magn Reson B 111: 209-219, 1996.

3. Berman JI, Berger MS, Mukherjee P and Henry RG: Diffusiontensor imaging-guided tracking of fibers of the pyramidal tract combined with intraoperative cortical stimulation mapping in patients with gliomas. J Neurosurg 101: 66-72, 2004.

4. Clark CA, Barrick TR, Murphy MM and Bell BA: White matter fiber tracking in patients with space-occupying lesions of the brain: a new technique for neurosurgical planning? Neuroimage 20: 1601-1608, 2003.

5. Henry RG, Berman JI, Nagarajan SS, Mukherjee P and Berger MS: Subcortical pathways serving cortical language sites: initial experience with diffusion tensor imaging fiber tracking combined with intraoperative language mapping. Neuroimage 21: 616-622, 2004.

6. Lazar M, Weinstein DM, Tsuruda JS, Hasan KM, Arfanakis K, Meyerand ME, Badie B, Rowley HA, Haughton V, Field A and Alexander AL: White matter tractography using diffusion tensor deflection. Hum Brain Mapp 18: 306-321, 2003.

7. Zeng $\mathrm{H}$ and Constable RT: Image distortion correction in EPI: comparison of field mapping with point spread function mapping. Magn Reson Med 48: 137-146, 2002.

8. Alexander AL, Tsuruda JS and Parker DL: Elimination of eddy current artifacts in diffusion-weighted echo planar images: the use of bipolar gradients. Magn Reson Med 38: 1016-1021, 1997.

9. Pipe JG: Motion correction with PROPELLER MRI: application to head motion and free-breathing cardiac imaging. Magn Reson Med 42: 963-969, 1999.

10. Pipe JG, Farthing VG and Forbes KP: Multishot diffusionweighted FSE using PROPELLER MRI. Magn Reson Med 47: 42-52, 2002.

11. Pipe JG: Turboprop: An improved PROPELLER sequence for diffusion weighted MRI. In: Proceedings of the ISMRM Tenth Scientific Meeting, Honolulu, Hawaii, p435, 2002.

12. Woods RP, Grafton ST, Holmes CJ, Cherry SR and Mazziotta JC: Automated image registration: I. General methods and intrasubject, intramodality validation. J Comp Assist Tomogr 22: 141-154, 1998.

13. Jezzard P and Balaban RS: Correction for geometric distortion in echo planar images from B0 field variations. Magn Reson Med 34: 65-73, 1995.

14. Andersson JL, Skare S and Ashburner J: How to correct susceptibility distortions in spin-echo echo-planar images: application to diffusion tensor imaging. Neuroimage 20: 870-888, 2003.

15. Cordes D, Arfanakis K, Haughton V and Meyerand ME: Geometric distortion correction in EPI using two images with orthogonal phase-encoding directions. In: Proceedings of the 8th Annual Meeting of ISMRM, Denver, CO, p1712, 2000.

16. Jezzard P, Barnett AS and Pierpaoli C: Characterization of and correction for eddy current artifacts in echo planar diffusion imaging. Magn Reson Med 39: 801-812, 1998.

17. Arfanakis K, Cordes D, Haughton VM, Carew JD and Meyerand ME: Independent component analysis applied to diffusion tensor MRI. Magn Reson Med 47: 354-363, 2002.

18. Arfanakis K, Tamhane AA, Pipe JG and Anastasio MA: k-space undersampling in PROPELLER imaging. Magn Reson Med 53: 675-683, 2005.

19. Pruessmann KP, Weiger M, Scheidegger MB and Boesiger P: SENSE: sensitivity encoding for fast MRI. Magn Reson Med 42: 952-962, 1999. 